\title{
Characterization of combinatorially independent permutation separability criteria
}

\author{
Paweł Wocjan ${ }^{1}$ and Michał Horodecki ${ }^{2}$ \\ 1 Computer Science Department $\&$ Institute for Quantum Information, \\ California Institute of Technology, Pasadena, CA 91125, USA \\ 2 Institute of Theoretical Physics and Astrophysics, University of Gdańsk, 80-952 Gdańsk, Poland
}

\begin{abstract}
The so-called permutation separability criteria are simple operational conditions that are necessary for separability of mixed states of multipartite systems: (1) permute the indices of the density matrix and (2) check if the trace norm of at least one of the resulting operators is greater than one. If it is greater than one then the state is necessarily entangled. A shortcoming of the permutation separability criteria is that many permutations give rise to dependent separability criteria. Therefore, we introduce a necessary condition for two permutations to yield independent criteria called combinatorial independence. This condition basically means that the map corresponding to one permutation cannot be obtained by concatenating the map corresponding to the second permutation with a norm-preserving map. We characterize completely combinatorially independent criteria, and determine simple permutations that represent all independent criteria. The representatives can be visualized by means of a simple graphical notation. They are composed of three basic operations: partial transpose, and two types of so-called reshufflings. In particular, for a four-partite system all criteria except one are composed of partial transpose and only one type of reshuffling; the exceptional one requires the second type of reshuffling. Furthermore, we show how to obtain efficiently for every permutation a simple representative. This method allows to check easily if two permutations are combinatorially equivalent or not.
\end{abstract}

\section{INTRODUCTION}

Entanglement theory has been actively developed for more than a decade. We know a lot about entanglement of bipartite and multipartite states, about how it can be manipulated and how it can be used as a resource for performing certain tasks in quantum information processing $\mathrm{ABH}^{+}$01]. However, so far one of the main problems of entanglement theory has not been resolved: the design of operational criteria allowing to detect whether a given state is entangled or not. Since the first treatments of this subject Wer89, HH94 a rich literature has been created (see e.g. $\mathrm{QIC}, \mathrm{BCH}^{+} 02, \mathrm{BDH}^{+} 02, \mathrm{MKB} 04, \mathrm{GL04}$, DPS04]).

For the purpose of this present paper, let us explicitly mention that a simple necessary and sufficient condition exists for qubit-qubit and qubit-qutrit systems, which is the partial transposition criterion Per96, HHH96. Subsequently, in CWY03 and Rud02 a new, equally simple criterion for bipartite systems was introduced, called or cross norm or realignment criterion. Though weaker in the case of two qubits, it turns out to be independent of the partial transpose in the case of two qutrits and higher dimensional systems. Then the multipartite case has been treated in [HHO2], where a family of permutation criteria was introduced, which present a generalization of both the partial transpose and the realignment criteria [1].

The basic idea underlying the permutation separability criteria is that the operators that are obtained by permuting the indices of the density matrix of any separable state still have trace norm not greater than one. If the norm is greater than one than the state is necessarily entangled. An advantage of the permutation criteria is that they are very easy to apply. However, some inconve- nience still remains, both for direct applications, as well as for gaining theoretical insight. The reason for this is that many different permutations can lead to the same separability criterion. Indeed, suppose that a given permutation $\sigma$ of indices does not change the norm of any operator (for example, because it gives rise to a unitary operation). Consider now some other permutation $\sigma^{\prime}$. Clearly, a permutation $\sigma^{\prime} \sigma$ where we first apply $\sigma^{\prime}$ and then $\sigma$, gives the same criterion as $\sigma^{\prime}$. Many permutation criteria are equivalent in this sense. We call this combinatorial (in)dependence. For bipartite systems one easily finds that as the only combinatorially independent permutation criteria one can choose partial transpose and realignment [HH02]. For multipartite systems the problem of determining independent criteria turns out to be more difficult. The problem of deriving independent criteria was attempted in Fan02] where combinatorially independent criteria for the tripartite case were determined and a method of deciding whether two criteria are equivalent was exhibited.

In this paper we solve the problem of characterizing combinatorially independent permutation criteria completely. For an $r$-partite system, we show that there are $\frac{1}{2}\left(\begin{array}{c}2 r \\ r\end{array}\right)-1$ combinatorially independent criteria. We provide a complete set of independent criteria, which are particularly simple. They can be built by combining three basic operations: partial transpose (acting on only one of the subsystems) and two kinds of so-called reshuffling (both acting on two of the subsystems). We introduce a simple graphical notation for the independent criteria and show how a general permutation can be brought into this form. As a result we obtain a simple and transparent method of verifying whether two permutations lead to equivalent criteria. In this way we have transformed the permutation criteria in a user-friendly tool, 
showing in a precise manner the structure of all combinatorially independent permutation separability criteria.

\section{DEFINITION OF PERMUTATION SEPARABILITY CRITERIA}

In this section we recall the definition and the properties of the permutation criteria for separability of multipartite mixed states. We consider states acting on $r$ subsystems whose Hilbert spaces have all the same dimension $d$. A general state $\rho$ on this joint Hilbert $\mathcal{H}$ space can be written as follows

$$
\sum_{i_{1}, i_{2}, \ldots, i_{2 r-1}, i_{2 r}} \rho_{i_{1} i_{2}, i_{3} i_{4}, \ldots, i_{2 r-1} i_{2 r}}\left|i_{1} i_{3} \ldots i_{2 r-1}\right\rangle\left\langle i_{2} i_{4} \ldots i_{2 r}\right|
$$

where all indices run from 1 to $d$, and the kets $\left|k_{1} \ldots k_{r}\right\rangle=\left|k_{1}\right\rangle \otimes\left|k_{2}\right\rangle \otimes \ldots \otimes\left|k_{r}\right\rangle$ denote the standard basis vectors in $\mathcal{H}$. Note that indices $i_{j}$ with odd subscripts correspond to rows of $\rho$ and those with even subscripts to columns of the density matrix.

Let $S_{2 r}$ denote the group of permutations of the set $\{1,2, \ldots, 2 r\}$. We define for each permutation $\sigma \in S_{2 r}$ a corresponding map $\Lambda_{\sigma}$ on operators acting on $\mathcal{H}$ by setting

$$
\begin{aligned}
& {\left[\Lambda_{\sigma}(\rho)\right]_{i_{1} i_{2}, i_{3} i_{4}, \ldots, i_{2 r-1} i_{2 r}} } \\
= & \rho_{i_{\sigma(1)} i_{\sigma(2)}, i_{\sigma(3)} i_{\sigma(4)}, \ldots, i_{\sigma(2 r-1)} i_{\sigma(r)}} .
\end{aligned}
$$

Note that the map $\sigma \mapsto \Lambda_{\sigma}$ is a homomorphism from the symmetric group $S_{2 r}$ into the group of invertible operators acting on $\mathcal{H}$, i.e., we have

$$
\left.\Lambda_{\sigma_{1} \sigma_{2}}(\rho)=\Lambda_{\sigma_{2}}\left(\Lambda_{\sigma_{1}}(\rho)\right)\right),
$$

where $\sigma_{1} \sigma_{2}$ denotes the composition of the permutations $\sigma_{1}$ and $\sigma_{2}$. Here and throughout the paper we use the convention that products of permutations are evaluated from left to right (i.e. $\sigma_{1}$ acts first, then $\sigma_{2}$ ).

According to [HHH02] each permutation $\sigma \in S_{2 r}$ of indices of $\rho$ gives rise to a corresponding permutation criterion of separability, as follows. It turns out that for separable states we have

$$
\left\|\Lambda_{\sigma}(\rho)\right\| \leq 1
$$

for all permutation $\sigma \in S_{2 r}$, where $\|A\|=\operatorname{Tr}\left(A A^{\dagger}\right)^{1 / 2}$ denotes the trace norm. Thus the above inequality constitutes a separability criterion. If the inequality is violated, it implies that the state $\rho$ is entangled.

\section{COMBINATORIAL INDEPENDENCE: A NECESSARY CONDITION FOR THE INDEPENDENCE OF THE PERMUTATION SEPARABILITY CRITERIA}

As already noted in the introduction, a major inconvenience in applying and understanding the permutation separability criteria stems from the fact that many permutations define trivial separability criteria or criteria that are not independent.

Let us first consider the case of trivial criteria. To do that, we introduce some notions. We call a map $\Lambda$ on operators acting on $\mathcal{H}$ norm-preserving if $\|\Lambda(\rho)\|=\|\rho\|$ for all operators $\rho$ on $\mathcal{H}$. Similarly, we say that a permutation $\sigma$ is norm-preserving if its corresponding map $\Lambda_{\sigma}$ is norm-preserving. Now, let $\sigma$ be a norm-preserving permutation. In this case $\left\|\Lambda_{\sigma}(\rho)\right\|=1$ for all quantum states $\rho$ and consequently $\sigma$ defines a trivial criterion. Such a criterion is useless because it cannot detect any entanglement.

For example, this can occur if the map $\Lambda_{\sigma}$ acts as

$$
\Lambda_{\sigma}(\rho)=U_{\sigma} \rho V_{\sigma}
$$

on all operators $\rho$ on $\mathcal{H}$, where $U_{\sigma}, V_{\sigma}$ are unitary transformations. This is because multiplication by unitary operators from the left or right cannot change the trace norm.

Another example for a trivial criterion is the transposition of the density matrix of the state $\rho$ (defined as the exchange of rows and columns). We call it global quantum transposition (GQT) in order to differentiate it from partial (quantum) transpositions and transpositions [2] It is readily verified that the permutation that gives rise to GQT is the following:

$$
\sigma=(1,2)(3,4) \cdots(2 r-1,2 r) .
$$

Let us now consider the problem of permutations leading to criteria that are not independent. Assume that two different permutations $\sigma$ and $\tau$ define two maps $\Lambda_{\sigma}$ and $\Lambda_{\tau}$ that are related by a norm-preserving map $\Lambda$. More precisely, assume that we have

$$
\Lambda_{\sigma}(\rho)=\Lambda\left(\Lambda_{\tau}(\rho)\right)
$$

for all operators $\rho$. It is clear that the separability criteria defined by $\sigma$ and $\tau$ are equivalent because

$$
\left\|\Lambda_{\sigma}(\rho)\right\|=\left\|\Lambda\left(\Lambda_{\tau}(\rho)\right)\right\|=\left\|\Lambda_{\tau}(\rho)\right\|
$$

for all quantum states. All entangled states that can be detected by $\sigma$ can also be detected by $\tau$ and vice versa.

Based on the above observations we define combinatorial independence of permutations that is a necessary condition for two permutations to yield independent separability criteria.

Definition 1 (Combinatorial independence). We say that a permutation $\sigma$ in $S_{2 r}$ (and its corresponding separability criterion) is trivial if the corresponding map $\Lambda_{\sigma}$ is norm-preserving. We say that two permutations $\sigma$ and $\tau$ in $S_{2 r}$ (and their corresponding separability criteria) are combinatorially independent if and only if there is no norm-preserving map $\Lambda$ on operators acting on $\mathcal{H}$ such that

$$
\Lambda_{\sigma}(\rho)=\Lambda\left(\Lambda_{\tau}(\rho)\right)
$$


for all operators $\rho$, i.e., the map $\Lambda_{\sigma}$ is the composition of $\Lambda_{\tau}$ with $\Lambda$ (where we apply first $\Lambda_{\tau}$ and then $\Lambda$ ).

In the following we derive a group-theoretical explanation of the combinatorial independence relation. The property in eq. (3) that the map $\sigma \mapsto \Lambda_{\sigma}$ is a homomorphism ensures that norm preserving permutations form a subgroup of $S_{2 r}$. This is because the composition of two norm-preserving permutations gives rise to the composition of two maps that do not change the trace norm. We call this group the group of norm-preserving permutations and denote it by $\mathcal{T}$.

Lemma 1 (Group of norm-preserving permutations). The group $\mathcal{T}$ of norm-preserving permutations is generated by transpositions exchanging only even points, by transpositions exchanging only odd points, and by the permutation realizing the global quantum transposition:

$\langle(2 k, 2 l),(2 k-1,2 l-1),(1,2)(3,4) \cdots(2 r-1,2 r)\rangle$,

where $1 \leq k<l \leq r$. The group $\mathcal{T}$ has the structure of a so-called wreath product: it contains two symmetric groups $S_{r}$ acting on even and odd numbers, respectively, and the subgroup $S_{2}$ generated by the permutation $(1,2)(3,4) \ldots(2 r-1,2 r)$ that switches between the two symmetric groups. The number of elements of the group $\mathcal{T}$ is equal to

$$
2 r ! r !
$$

Here the factor 2 accounts for the permutation $(1,2)(3,4) \cdots(2 r-1,2 r)$, and both $r$ ! factors for the permutations groups on even and odd points.

Proof. The proof consists of two steps. We determine the group structure by showing (1) that the group generated in eq. (9) is a subgroup of the group of normpreserving permutations and (2) that all permutations that are not contained in this subgroup change the trace norm of some quantum states. Our graphical notation is decisive for the proof the second part. It is postponed to the next section where it follows readily as a corollary from Theorem 2

We have already seen that the permutation $(1,2)(3,4) \cdots(2 r-1,2 r)$ is norm-preserving. It remains to be shown that the odd permutations $(2 k-1,2 l-1)$ and the even permutations $(2 k, 2 l)$ are norm-preserving. To see that we show that their corresponding maps can be realized by multiplication with unitary operators $V_{k l}$ from left and right, respectively. Let $V$ denote the swap operator acting on $\mathbb{C}^{d} \otimes \mathbb{C}^{d}$, i.e.,

$$
V=\sum_{i j=1}^{d}|i j\rangle\langle j i|
$$

The swap operator has the property $V(|\psi\rangle \otimes|\phi\rangle)=$ $|\phi\rangle \otimes|\psi\rangle$ for any $|\phi\rangle,|\psi\rangle \in \mathbb{C}^{d}$. The swap operators between any two subsystems of $\mathcal{H}$ are defined by embedding
$V$ suitably, i.e., the operators $V_{k l}$ act as the swap operator $V$ on the subsystems $k$ and $l$ and as the identity on the remaining subsystems. Let us define two maps on operators on $\mathcal{H}$ with the help of the swap operators:

$$
V_{k l}^{L}(\rho)=V_{k l} \rho, \quad V_{k l}^{R}(\rho)=\rho V_{k l} .
$$

Note that the above maps on operators come from transpositions:

$$
V_{k l}^{R}=\Lambda_{\tau}, \quad V_{k l}^{L}=\Lambda_{\tau^{\prime}}
$$

where $\tau=(2 k-1,2 l-1)$ and $\tau^{\prime}=(2 k, 2 l)$. For this reason the transpositions exchanging two even or two odd numbers are norm-preserving and therefore constitute trivial separability criteria. Since compositions of norm-preserving permutations are again norm-preserving permutations we see that all permutations of odd numbers (and even numbers) are trivial permutations (this is because transpositions generate all permutations). The wreath product structure follows from the fact that if we multiply the permutations that generate $\mathcal{T}$ we can always propagate the permutations realizing the GQT to the right. Thus any element of the group can be see as either a composition of some permutation of even indices and some permutation of odd indices, or such a composition followed by GQT. Thus the number of elements is $2 r ! r !$.

Lemma 2 (Group-theoretic structure of combinatorial independence). The combinatorially independent permutation criteria correspond to the right cosets $S_{2 r} / \mathcal{T}$ of $\mathcal{T}$ in $S_{2 r}$. The number of different cosets is

$$
\frac{1}{2}\left(\begin{array}{c}
2 r \\
r
\end{array}\right) .
$$

(The trivial criteria correspond to the coset defined by $\mathcal{T}$.)

Proof. Let $\sigma$ and $\tau$ be two permutations that are combinatorially dependent, i.e., there is a norm-preserving map $\Lambda$ such that $\Lambda_{\sigma}(\rho)=\Lambda\left(\Lambda_{\tau}(\rho)\right)$ for all operators $\rho$. This is equivalent to $\Lambda=\Lambda_{\tau^{-1} \sigma}$ and consequently $\tau^{-1} \sigma$ must be a norm-preserving permutation.

Therefore, for any given permutation $\sigma$, all permutations belonging to the right coset defined by $\sigma$, i.e., all permutation in the set $\{\sigma \tau: \tau \in \mathcal{T}\}$ are combinatorially dependent. This shows that the number of combinatorially independent criteria is equal to the number of different right cosets $S_{2 r} / \mathcal{T}$. It is given by

$$
\left|S_{2 r} / \mathcal{T}\right|=\frac{\left|S_{2 r}\right|}{|\mathcal{T}|}=\frac{(2 r) !}{2 r ! r !}=\frac{1}{2}\left(\begin{array}{c}
2 r \\
r
\end{array}\right) .
$$

This ends the proof.

One of the cosets corresponds to the group $\mathcal{T}$ of normpreserving permutations, so that we get $\frac{1}{2}\left(\begin{array}{c}2 r \\ r\end{array}\right)-1$ nontrivial combinatorially independent permutation separability criteria. 


\begin{tabular}{c|c|c} 
graphical representation & corresponding permutation & name \\
\hline \hline$k \bullet \longrightarrow \bullet l$ & $(2 k, 2 l-1)$ & reshuffle $R_{k l}$ \\
\hline$k \bullet \longleftarrow \bullet l$ & $(2 k-1,2 l)$ & reshuffle $R_{l k}^{\prime}$ \\
\hline$k \circlearrowright$ & $(2 k-1,2 k)$ & partial transpose \\
\hline$k \bullet$ & () & identity
\end{tabular}

FIG. 1: Basic permutations

\begin{tabular}{c|c} 
valid configurations & invalid configurations \\
\hline \hline$\bullet \longrightarrow \bullet \longrightarrow \bullet$ & $\bullet \longleftarrow \bullet \longrightarrow \bullet$ \\
\hline$\bullet \longleftarrow \bullet \longleftarrow \bullet$ & $\bullet \longrightarrow \bullet \longleftarrow \bullet$ \\
\hline$\circlearrowright \quad \bullet \longrightarrow \bullet$ & $\circlearrowright \longleftarrow \bullet \bullet \bullet$ \\
\hline$\circlearrowright \quad \circlearrowright \quad \bullet$ & $\circlearrowright \longrightarrow \bullet \longrightarrow \bullet$
\end{tabular}

FIG. 2: Examples for some valid and invalid configurations of arrows for three subsystems

\section{SIMPLE REPRESENTATIVES OF COMBINATORIALLY INDEPENDENT CRITERIA}

Of course, one could use a computer algebra system to determine the combinatorially independent criteria. However in such a way, one would obtain some more or less arbitrary representatives for the right cosets $S_{2 r} / \mathcal{T}$, which do not give any insight into the structure of the permutation criteria. Therefore, we determine especially simple representatives for the right cosets. To this end, we develop a graphical notation for involutions, i.e., permutations that can be written as a product of disjoint transpositions. The notation is very helpful in deriving the representatives. The graphical notation is presented in fig. 1] The basic permutations are identity, partial transpose, and two types of reshuffling ZB04].

For an arrow $[k \bullet \longrightarrow \bullet l]$ we say that $k$ is its tail and $l$ is its head. We call an arrow of the form $\left[\begin{array}{ll}k & \circlearrowright\end{array}\right]$ a loop. For such a loop we say that $k$ is both its head and its tail. The support of an arrow is the set containing its head and tail. Let $C$ be a configuration of arrows. We say that $C$ is disjoint if the supports of all pairs of arrows are disjoint. Intuitively, this means that the arrows do not touch each other.

Theorem 1 (Representation by disjoint configurations). The right cosets $S_{2 r} / \mathcal{T}$ can be always represented by disjoint configurations of arrows.

To prove this theorem we will apply three basic transformations on permutations called pruning, chopping and exchanging heads. These transformations can be realized by multiplying the permutations with elements of the group $\mathcal{T}$ from the right. The application of these three transformations will bring every permutation into its normal form, i.e., an equivalent permutation that can be represented by disjoint configurations of arrows. As an intermediary step we will need to work with configurations of arrows that are not necessarily disjoint. It is important that some non-disjoint configurations of arrows should not be allowed because they do not determine permutations unambiguously. Two arrows that point to the same subsystem do not define a permutation unambiguously because their corresponding transpositions are not disjoint, i.e., they do not commute and it would be unclear which transposition should be applied first. Similarly, two arrows starting from the same subsystem or an arrow starting from or pointing toward a loop are not unambiguous. For these reasons, these configurations are not allowed. Some of these situations are shown in fig. 2 In the following all configurations we will use will be valid.

Rule 1 (Pruning). Let $\sigma \in S_{2 r}$ be any permutation. Then we can prune all cycles of $\sigma$ such that there are no adjacent even or odd numbers. The pruning can be achieved by multiplication by a norm-preserving permutation from the right.

Proof. Write $\sigma$ as a product of disjoint cycles. Consider any cycle, with two adjacent numbers $n_{1}$ and $n_{2}$ :

$$
\left(k_{1}, \ldots, k_{s}, n_{1}, n_{2}, l_{1}, \ldots, l_{s^{\prime}}\right)
$$

We note that by applying the permutation $\left(n_{1}, n_{2}\right)$ from the right we get:

$$
\begin{aligned}
& \left(k_{1}, \ldots, k_{s}, n_{1}, n_{2}, l_{1}, \ldots, l_{s^{\prime}}\right)\left(n_{1}, n_{2}\right) \\
= & \left(k_{1}, \ldots, k_{s}, n_{2}, l_{1}, \ldots, l_{s^{\prime}}\right)\left(n_{1}\right)
\end{aligned}
$$

Now, if $n_{1}$ and $n_{2}$ are either both odd or both even, then the permutation $\left(n_{1}, n_{2}\right)$ is a norm-preserving permutation. Thus the initial cycle is equivalent to the cycle with $n_{1}$ removed. Note that the permutation does not affect the other cycles because the cycles are disjoint. Therefore, the pruning process can be applied independently to the cycles.

Rule 2 (Chopping). Let $\sigma \in S_{2 r}$ be any permutation that cannot be further simplified with the help of the pruning rule. Then we can chop $\sigma$ into a product of disjoint transpositions, i.e., a permutation that can be represented by a valid configuration of arrows. 
A)

B)
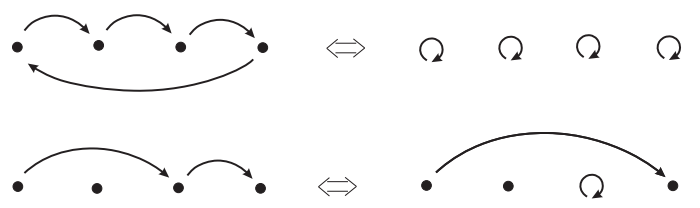

C)

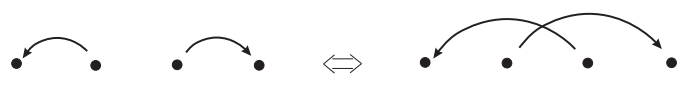

FIG. 3: Exchanging heads.

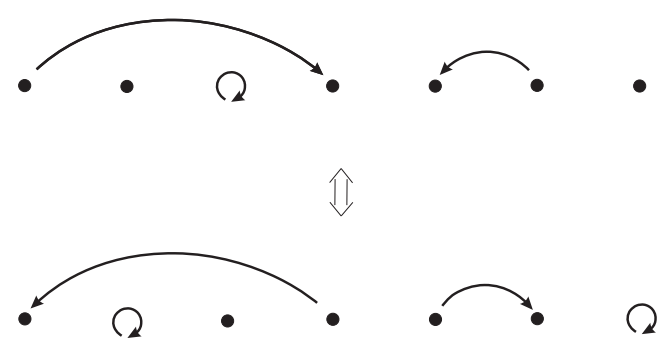

FIG. 4: Example for the change under flipping.

\section{Proof. Let}

$$
\left(n_{1}, p_{1}, n_{2}, p_{2}, n_{3}, p_{3}, \ldots, n_{k}, p_{k}\right)
$$

be any cycle of $\sigma$. If we apply the permutation $\left(n_{2}, n_{1}\right)\left(n_{3}, n_{2}\right) \ldots\left(n_{1}, n_{k}\right)$ to the above cycle then we obtain:

$$
\begin{aligned}
& \left(n_{1}, p_{1}, \ldots, n_{k}, p_{k}\right)\left(n_{2}, n_{1}\right)\left(n_{3}, n_{2}\right) \ldots\left(n_{1}, n_{k}\right) \\
= & \left(n_{1}, p_{1}\right)\left(n_{2}, p_{2}\right) \ldots\left(n_{k}, p_{k}\right)
\end{aligned}
$$

Now, if the $n_{i}$ 's are either all even or all odd, then the applied permutation $\left(n_{2}, n_{1}\right)\left(n_{3}, n_{2}\right) \ldots\left(n_{1}, n_{k}\right)$ is normpreserving.

After applying the chopping rule we end up with a permutation that can be written as a product of disjoint transpositions. Therefore, the resulting permutation can represented as a valid configuration of arrows.

Rule 3 (Exchanging heads). Let $C$ be a configuration of arrows. Then by exchanging the heads of the arrows we obtain an equivalent disjoint configuration of arrows.

Proof. For arrows defined by $(2 k, 2 l-1)(2 m, 2 n-1)$ we take the norm-preserving permutation $(2 l-1,2 n-$ $1)(2 k, 2 m)$. It is easily verified that the operation produces arrows with exchanged heads. Note that this works if one or two of the arrows are loops. Some examples are presented in fig. 3.

Before proving Theorem 1 we will provide yet another rule, which is not needed in the proof of theorem, but will be needed later. We say that the subsystem $k$ is free in a valid disjoint configuration if it is not contained in the support of any arrow of $C$. In other words, a subsystem is free, when it is neither head nor tail of any arrow (including a loop).

Rule 4 (Flipping). Let $C$ be a disjoint configuration. If we apply first the global quantum transposition and then the norm-preserving permutation

$$
\prod_{i=1}^{a}\left(2 t_{i}-1,2 h_{i}-1\right)\left(2 t_{i}, 2 h_{i}\right)
$$

where $t_{1} \rightarrow h_{1}, \ldots, t_{a} \rightarrow h_{a}$ are all arrows of $C$ that are not loops (i.e., $t_{i} \neq h_{i}$ ), then $C$ is changed as follows: the directions of all arrows that are not loops are reversed, all loops are removed, and new loops are created on all free subsystems.

Proof. Let $k$ be a free subsystem of $C$. Then we obtain $(2 k-1,2 k)$ on subsystem $k$ by multiplying with GQT. Similarly, if a subsystem $l$ has a loop, then the corresponding permutation $(2 l-1,2 l)$ is removed by multiplying with GQT.

Let $t \rightarrow h$ be an arrow of $C$ with $t \neq h$. Then the corresponding permutation $(2 t, 2 h-1)$ is changed by multiplying with GQT as follows

$$
\begin{aligned}
& (2 t, 2 h-1)(2 t-1,2 t)(2 h-1,2 h) \\
= & (2 t, 2 h, 2 h-1,2 t-1) .
\end{aligned}
$$

Observe that there is a pair of adjacent even numbers and a pair of adjacent odd numbers. Therefore, we can 


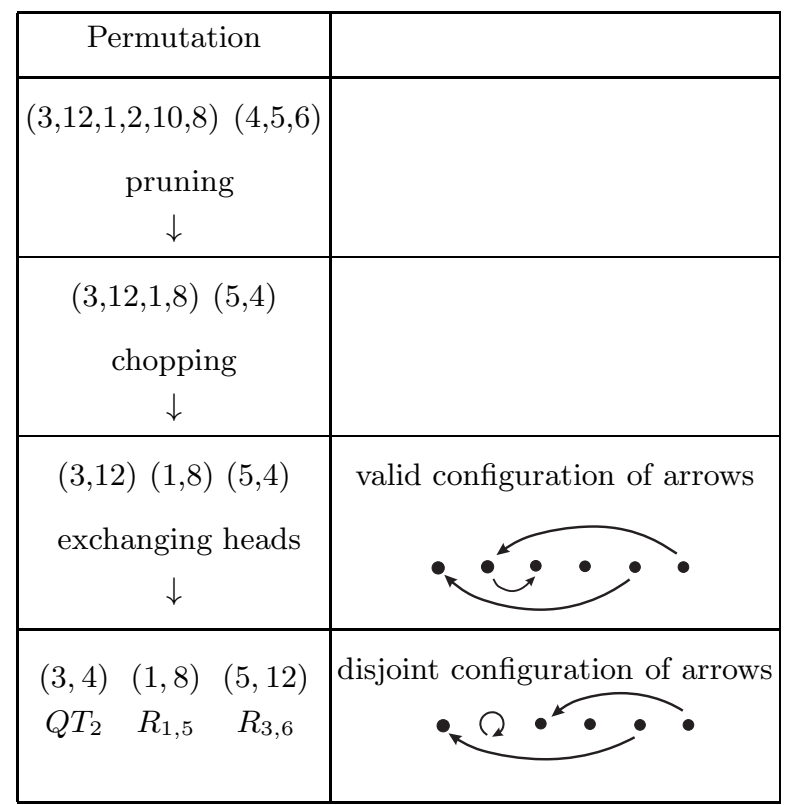

TABLE I: Steps of our algorithm in Theorem [

apply the pruning rule. This is realized by multiplying with $(2 t-1,2 h-1)(2 t, 2 h)$. We obtain

$$
\begin{aligned}
& (2 t, 2 h, 2 h-1,2 t-1)(2 t-1,2 h-1)(2 t, 2 h) \\
= & (2 h, 2 t-1) .
\end{aligned}
$$

The resulting permutation $(2 h, 2 t-1)$ is represented by the arrow $t \leftarrow h$. This completes the proof.

The action of flipping is shown for a disjoint arrow configuration in fig. [4

Now, let us prove Theorem 1

Proof of Theorem 1 Consider an arbitrary permutation. Perform first the following steps: (1) decompose the permutation into disjoint cycles, (2) apply pruning, and (3) apply chopping.

After applying these three rules we have transformed the permutation into an equivalent one that can be represented by a (valid) configurations of arrows. Observe that configurations of arrows consists of three types of separate objects: closed paths of arrows, open paths of arrows, loops, and free subsystems. Now we apply the rule exchanging heads to paths of arrows. By doing this suitably all closed paths can be changed into a collection of loops (see fig. [3 A), and all open paths into loops plus one arrow - the one going from the end to the start of the open path (see fig. 3 B). In this way, collections of all paths are changed into sets of disjoint arrows or loops. This completes the proof.

An example illustrating the steps of our algorithm for transforming permutations to their corresponding disjoint arrow configurations in shown in table 【.

Note that after carrying out the transformation described above we can (optionally) apply the flipping op- eration, if there are too many loops. But more interestingly, the application of flipping, though not needed, makes the representation much nicer. Namely, one sees that the transformations of exchanging heads used in the proof of Theorem 1 plus flipping act on any valid configuration (hence not necessarily the disjoint one) as follows:

1. every closed path of arrows disappears

2. every open path of arrows is replaced by its closing arrow (i.e., the arrow that was missing to make the path closed)

3. every loop disappears

4. every free subsystem acquires a loop

Of course in the case of disjoint configurations, the closed paths do not occur, so that the item 1 will never be used; also the item 2 will mean changing direction of an arrow.

As a corollary of Theorem 1 we obtain that the group generated in eq. (9) is indeed the group of normpreserving permutations (this is the second part of Lemma 1 whose proof was postponed).

Corollary 1. The group $\mathcal{T}$ of norm-preserving permutations is equal to the group $\mathcal{T}^{\prime}$ generated by

$$
(2 k, 2 l),(2 k-1,2 l-1),(1,2)(3,4) \cdots(2 r-1,2 r),
$$

where $1 \leq k<l \leq r$.

Proof. We already know that $\mathcal{T}^{\prime} \subset \mathcal{T}$. Theorem 1 shows that all permutations can be represented by disjoint configurations. Obviously, all permutations in $\mathcal{T}^{\prime}$ can be represented by the empty configuration (i.e. the one representing the trivial permutation - the identity). Therefore, a permutation $\sigma \notin \mathcal{T}^{\prime}$ cannot be represented by the empty configuration. Without loss of generality $\sigma$ can be represented by a disjoint configuration $C$ such that (1) $C$ contains an arrow from $k$ to $l$ or (2) $C$ contains a loop on $k$ and a free subsystem $l$ for some $k \neq l$. Now, known results for the bipartite case imply that there are entangled states detectable by the arrow (corresponding to realignment) in (1) and the loop and the free subsystem (corresponding to the partial transpose) in (2). This shows that all permutations not contained in $\mathcal{T}^{\prime}$ change the norm of some quantum states. Therefore, $\mathcal{T}^{\prime}$ is equal to $\mathcal{T}$.

Let us introduce the following notation. Let $H$ and $T$ be the head and tail sets of a disjoint configuration $C$. Denote by $\bar{H}$ and $\bar{T}$ the head and tail sets of the configuration $\bar{C}$ that is obtained from $C$ by applying the global quantum transposition. Fig. [5 shows an example of how the head and tail sets are changed by flipping. We write $(H, T) \neq\left(H^{\prime}, T^{\prime}\right)$ to denote that $H \neq H^{\prime}$ or $T \neq T^{\prime}$.

Theorem 2 (Characterization of combinatorially independent permutation separability criteria). 


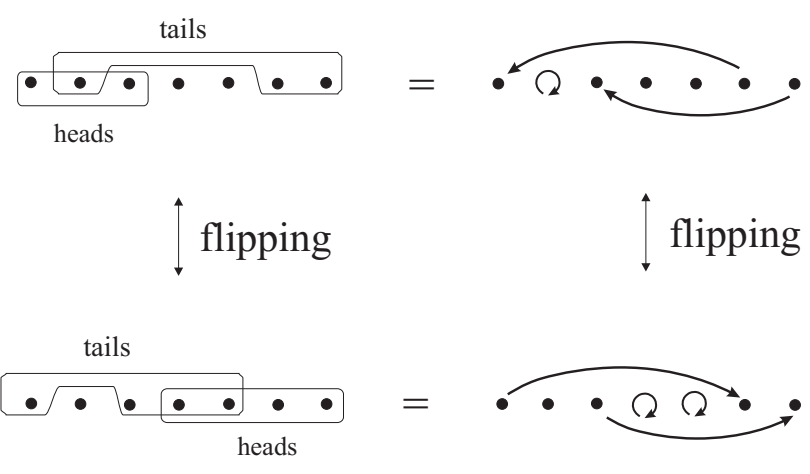

FIG. 5: Head and tail sets of two criteria related by the rule flipping.

All combinatorially independent separability criteria (or equivalently all right cosets $S_{2 r} / \mathcal{T}$ ) can be represented by a collection of head sets $H_{i}$ and tail sets $T_{i}$ satisfying the properties:

$$
\begin{aligned}
& \text { 1. }\left(H_{k}, T_{k}\right) \neq\left(H_{l}, T_{l}\right) \\
& \text { 2. }\left(H_{k}, T_{k}\right) \neq\left(\bar{H}_{l}, \bar{T}_{l}\right)
\end{aligned}
$$

for all $k \neq l$.

Thus, to choose combinatorially independent criteria, we first consider all possible choices of tails and heads (of course, in each choice, the number of heads must be equal to the number of tails). Then we are almost done: the criteria we have obtained are pairwise equivalent, i.e., to each criterion there is exactly one other equivalent criterion - the one related by flipping. Now, to get a set of independent criteria, we keep only one criterion from each pair.

Proof of Theorem 2 Due to Theorem 1 we know that all combinatorially independent criteria can be represented by disjoint configurations. The first condition is necessary because all configurations with equal head and tail sets can be transformed into each other using the rule exchanging heads. The second condition is necessary because then configurations can be obtained by flipping.

Now we count the number of pairs of head and tail sets satisfying the above conditions and show that it is equal to the number $\left|S_{2 r} / \mathcal{T}\right|$ of right cosets $S_{2 r} / \mathcal{T}$. Therefore, the above conditions fully characterize the combinatorially independent criteria.

The number of pairs of head and tail sets inequivalent with respect to the first condition is

$$
\sum_{k=0}^{r}\left(\begin{array}{l}
r \\
k
\end{array}\right)\left(\begin{array}{l}
r \\
k
\end{array}\right)=\left(\begin{array}{c}
2 r \\
r
\end{array}\right)
$$

The summation index $k$ corresponds to the number of arrows in the configuration. The first factorial is the number of possibilities of choosing $k$ heads and the second of choosing $k$ tails. The equality in (20) is a standard identity of binomial coefficients. If we allow the second rule then must divide the binomial coefficient $\left(\begin{array}{c}2 r \\ r\end{array}\right)$ by 2 . But this is the number of different cosets $S_{2 r} / \mathcal{T}$.

\section{EXAMPLES OF COMBINATORIALLY INDEPENDENT CRITERIA}

In this section we present all combinatorially independent permutation criteria for two, three and four particles. The arrow notation we have used so far is convenient for singling out combinatorially independent criteria. However, the arrow notation is based on a linear ordering of the particles, which is not convenient for visualizing the chosen independent criteria.

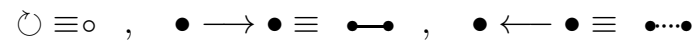

The criteria are visualized in fig. 6 For three particles, all criteria are combinations of reshuffling $R$ and partial transpose. For four particles a new possibility appears, which is compatible with Ref. Fan02]. We see that only one criterion uses different reshuffling $R^{\prime}$. Conversely, the application of GQT would lead to a complementary set of criteria, where only one reshuffling $R$ would be present.

\section{CONCLUDING REMARKS}

We have analyzed and simplified the structure of the permutation criteria. To this end, we have introduced the notion of combinatorial independence that is necessary for two permutation to yield independent separability criteria and completely characterized all combinatorially independent permutation criteria. The especially simple form of our representatives of all combinatorially independent criteria show explicitly how the permutation criteria probe the quantum state: to use the full power of permutation criteria, we can restrict ourselves to simple building blocks given by partial transpose and two types of reshuffling. This could be the starting point toward fully understanding the power of the permutation separability criteria, i.e., determining if there are entangled states that cannot be detected by any permutation criterion and establishing which permutations define truly independent separability criteria. In particular, our results simplify the evaluation of candidates for the entanglement measure proposed in $\mathrm{HHH} 02$. Perhaps our tools could enable a qualitative classification of multipartite entanglement with respect to some combinatorial classes.

Acknowledgments. We would like to thank Asa Ericsson and Lieven Clarisse for useful comments. The paper has been written while M. H. was visiting the Institute for Quantum Information, California Institute of Technology. M. H. is supported by Polish Ministry of Scientific Research and Information Technology under 
a)

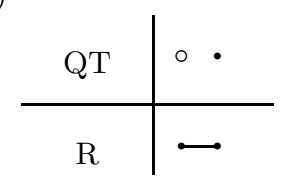

b)

\begin{tabular}{c|lll}
$\mathrm{QT}$ & $\circ$ & $\bullet$ & $\cdot$ \\
$\mathrm{R}$ & \rfloor$^{\cdot}$ & $\bullet$ & $\cdot$ \\
\hline $\mathrm{R}+\mathrm{QT}$ & \rfloor$_{\circ}$ & $\circ$ & $\circ$
\end{tabular}

c)

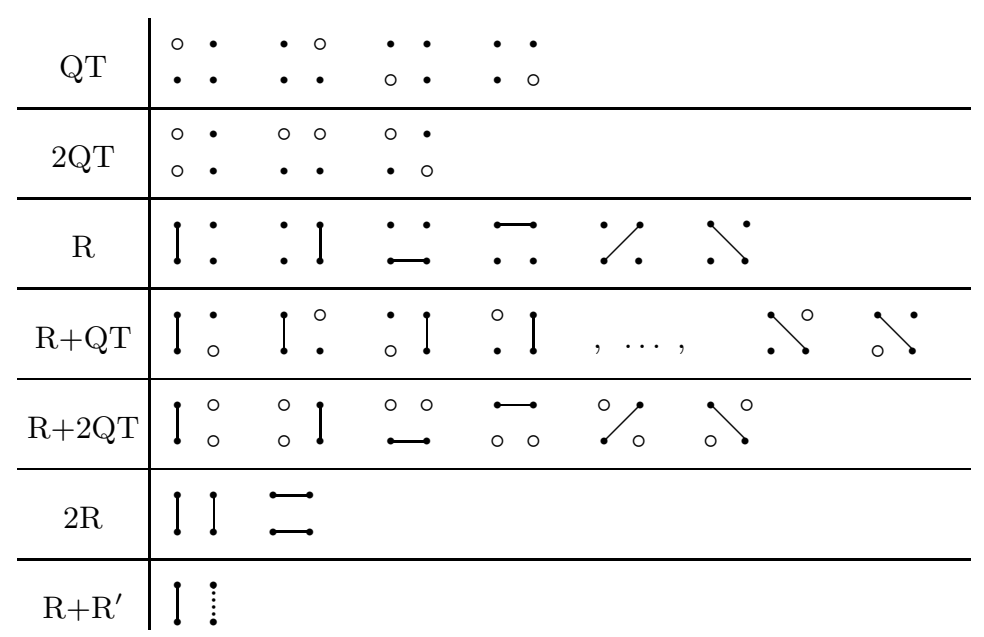

FIG. 6: Combinatorially independent permutation criteria for a) two, b) three, and c) four particles. Bars represent reshuffling $R$ and open circles represent quantum transpositions. The dotted line in c) represents the second type of reshuffling $R^{\prime}$.

the (solicited) grant no. PBZ-MIN-008/P03/2003 and by EC grants RESQ, contract no. IST-2001-37559 and QUPRODIS, contract no. IST-2001-38877. P.W. is sup- ported by the National Science Foundation under the grant no. EIA 0086038.
$\left[\mathrm{ABH}^{+} 01\right]$ Gernot Alber, Thomas Beth, Michał Horodecki, Paweł Horodecki, Ryszard Horodecki, Martin Rötteler, Harald Weinfurter, Reinhard Werner, and Anton Zeilinger. Quantum Information: An Introduction to Basic Theoretical Concepts and Experiments. Springer, 2001.

$\left[\mathrm{BCH}^{+} 02\right]$ Dagmar Bruss, J. I. Cirac, Paweł Horodecki, F. Hulpke, Barbara Kraus, Maciej Lewenstein, and Anna Sanpera. Reflections upon separability and distillability. J. Mod. Opt., 49:1399-1418, 2002. quant-ph/0110081

$\left[\mathrm{BDH}^{+} 02\right]$ Piotr Badziag, Piotr Deuar, Michal Horodecki, Pawel Horodecki, and Ryszard Horodecki. Concurrence in arbitrary dimensions. J. Mod. Opt., 49:1289-1297, 2002. quant-ph/0107147

[CW02] Kai Chen and Ling-An Wu. The generalized partial transposition criterion for separability of multipartite quantum states. Phys. Lett. A, 306:14-20, 2002. quant-ph/0208058
[CWY03] Kai Chen, Ling-An Wu, and Li Yang. A matrix realignment method for recognizing entanglement. quant-ph/0205017, 2003.

[DPS04] Andrew C. Doherty, Pablo A. Parrilo, and Federico M. Spedalieri. Detecting multipartite entanglement. quant-ph/0407143, 2004.

[Fan02] Heng Fan. A note on separability criteria for multipartite state. quant-ph/0210168, 2002.

[GL04] Otfried Guehne and Maciej Lewenstein. Separability criteria from uncertainty relations. AIP Conf. Proc., 734:230, 2004. quant-ph/0409140

[HH94] Paweł Horodecki and Ryszard Horodecki. Quantum redundancies and local realism. Phys. Lett. A, 194:147$152,1994$.

[HHH96] Michał Horodecki, Paweł Horodecki, and Ryszard Horodecki. Separability of mixed states: Necessary and sufficient conditions. Phys. Lett. A, 223:1, 1996. 
quant-ph/9605038

[HHH02] Michal Horodecki, Pawel Horodecki, and Ryszard Horodecki. Separability of mixed quantum states: linear contractions approach. quant-ph/0206008, 2002.

[MKB04] Florian Mintert, Marek Kus, and Andreas Buchleitner. Concurrence of mixed bipartite quantum states in arbitrary dimensions. Phys. Rev. Lett, 92:167902, 2004. quant-ph/0403063

[Per96] Asher Peres. Separability criterion for density matrices. Phys. Rev. Lett., 77:1413, 1996.

[QIC] First volume of Quantum Information \& Computation, 2001.

[Rud02] Olivier Rudolph. Further results on the cross norm criterion for separability. quant-ph/0202121 2002.

[Wer89] Reinhard Werner. Quantum states with EinsteinRosen-Podolsky correlations admitting a hidden-variable model. Phys. Rev., A 40:4277, 1989.

[ZB04] Karol Zyczkowski and Ingemar Bengtsson. On duality between quantum maps and quantum states. Open Sys. Inf. Dyn., 11:3-42, 2004. quant-ph/0401119

[1] Another generalization for multipartite states was introduced in [CW02].

[2] We mean by a transposition a permutation of the form $(a, b)$ and by a quantum transposition the transpose of a matrix 

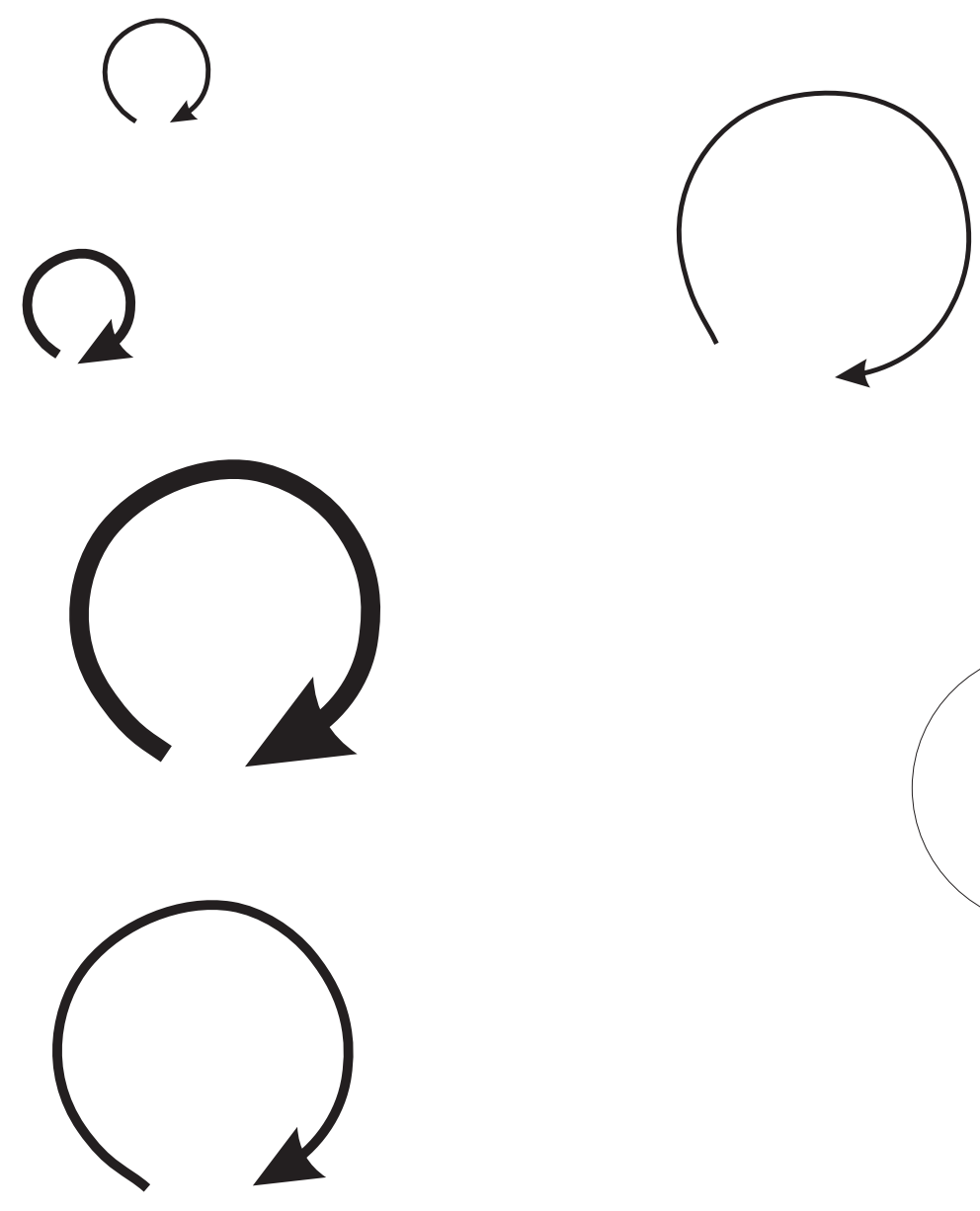